\title{
Multicriteria Feasibility Evaluation for Rural Transit in Georgia
}

George D. Mazur, Cambridge Systematics, Inc. Karen K. Dixon, Georgia Institute of Technology

Wayne A. Sarasua, Clemson University

\section{Abstract}

This article describes transit feasibility analysis features of the Georgia Department of Transportation's (GDOT's) Multimodal Transportation Planning Tool (MTPT). Using open databases that are available agencywide, the MTPT can provide a system-level analysis of transportation requirements of rural areas, identify potential implementation constraints early in the planning process, and develop a prioritized project list by mode for an analysis region. In addition to demand-responsive transit, the MTPT addresses intercity bus, intercity passenger rail, commuter rail, highways, aviation, and bicycle modes. This article focuses on the demand-responsive transit component of the MTPT, and provides an overview of how the MTPT can help automate system-level transit planning for the general public in rural Georgia.

The MTPT transit analysis assesses service feasibility for nonurbanized areas that do not currently have local transit service for the general public. In the MTPT, transit service feasibility considers the existence of human service transit providers in the county; the percentage of population in certain "target" populations; and estimates of potential ridership, vehicle requirements, capital costs, operating costs, and economic benefits. The first factor indicates if opportunities may exist to coordinate service with existing providers, and helps to address funding issues. The second factor assesses market characteristics of the target area as one determinant of potential service need. The third factor provides a range of potential values for key operating and 
financial statistics at a system-planning level of analysis. The intent of the analysis is to identify those locations where it makes sense to more seriously analyze new demandresponsive services.

This article should be helpful for transportation planners with responsibility for developing system-level transportation plans and programs at the county, region, and state levels. It will also be helpful for decision-makers who are trying to match transit funding to areas with high service needs.

\section{Introduction}

The passage of the Intermodal Surface Transportation Efficiency Act (ISTEA) and the Transportation Equity Act for the 21st Century (TEA-21) brought greater emphasis to congestion mitigation and multimodal planning in transportation. At the outset of the "ISTEA era," state departments of transportation (DOTs) committed significant resources to those metropolitan areas and rapidly developing fringe regions where congestion and intermodal problems were most prevalent. DOTs placed relatively less planning emphasis and resources on rural transportation issues, particularly for nonauto transportation modes such as transit services. Further, while statewide planning issues have garnered increasing research funds, few DOTs have undertaken research efforts to develop new ways to assist planners in identifying and prioritizing systemlevel needs in rural areas (Federal Highway Administration 1995).

System-level evaluation of transit needs and opportunities have traditionally presented challenges to transportation planners. Many of the available techniques have been refined for corridor- or route-level planning. Current system-level approaches to transit and multimodal planning borrow strategies from traditional highway planning with heavy emphasis on demand forecasting. However, considerations such as market characteristics and the unique financing structure of transit capital and operations suggest that a broader approach to system-level transit planning may be suitable. This broader approach is particularly warranted for statewide or rural applications, where local transit can be dominated by demand-responsive services that do not lend themselves to evaluation by techniques more suited for fixed-guideway or fixed-route bus services. 
Since passage of the Statewide Transportation Plan in 1994, the GDOT has been committed to achieving "a new vision for transportation in Georgia" that supports economic development, provides mobility options, enhances the quality of life, and allows full participation in the planning process (GDOT 1994). One primary recommendation from the statewide plan was to reorient the planning processes throughout the State to facilitate multimodal planning, programming, and decision making.

In the fall of 1994, GDOT's Office of Planning initiated development of a computer-based planning tool to aid in evaluating the multimodal transportation requirements of rural areas, including needs related to demand-responsive transit. This article reviews the application of GDOT's MTPT for system-level analysis of rural transit needs for the general public (i.e., "nonprogram" transit services). The article begins with a brief overview of MTPT capabilities. It then discusses various planning options for rural transit, as well as their integration into the MTPT. Finally, the article highlights steps taken to validate the analysis modules and potential steps for further enhancement.

\section{The Multimodal Transportation Planning Tool}

The MTPT is a comprehensive system- and project-level planning tool that can be used to identify needs and project priorities for nonurbanized areas in Georgia (Dixon et al. 2000; Mazur and Sarasua 1997; Mazur et al. 1996). The MTPT integrates a series of analytical routines into a Geographic Information System (GIS) platform, allowing an assessment of passenger travel on essentially all surface transportation modes.

At the system level, the tool can assess various metrics of current and future operational and physical performance, and identify those modal elements that fail to meet threshold values or that have been previously identified as modal needs. At the project level, the tool can perform a more detailed safety, operational, and physical analysis of roadway corridors, including those targeted for statewide bicycle routes. The MTPT can produce results at various aggregation levels including individual corridors, cities, counties, and GDOT administrative districts. In a typical session, an MTPT user identifies the geographic area to analyze, identifies modes to analyze, enters any user-specified parameters, conducts baseline and sensitivity analyses, and displays the results. 
The MTPT operates through an interface with several existing GDOT databases, allowing for querying and analysis of up-to-date physical and operational attributes. For demand-responsive transit, the MTPT relies on several internal databases that describe rural transit services in Georgia:

1)a county-level socioeconomic database containing detailed 1990 census data, population totals for 1960 to 1990 , and land area;

2)two service provider databases that list existing "program" and "nonprogram" rural transit providers; and

3)an urbanized county database that lists counties that are wholly or partially urbanized.

These databases provide the information needed to run routines developed specifically for the MTPT. The transit databases, together with a road characteristic database, GIS road maps, modal project data, supplemental databases (crash history, bicycle routes, etc.), and the system software, are written to a single compact disk, allowing the program to be easily shared with agencies throughout the State. This functionality is expanded by the MTPT's development for use with multiple operating systems on a personal computer.

\section{Rural Transit Services}

As with urban transit services, system-level planning for rural transit generally involves considerations of market feasibility, service need, potential usage, cost, and finance issues. However, the relative priority of the issues, methods used to assess the issues, and underlying scale of investment are typically different in urban and rural areas. While large-scale computerized travel-demand models are typically a central planning feature for large urban transit investments, use of data-intensive modeling efforts has historically not been an option for rural transit services.

In the past, several authors have suggested that comprehensive, longrange planning with travel-demand models be deemphasized in rural areas in favor of short-range corridor- and project-level planning (Fleet 1978; Golenburg 1978; Litz 1980; Smith 1982; Stover 1978). With more recent advances in computing technology, areas have begun to develop broad-scale modeling applications for rural transit (Black 1993; Attaluri et al. 1997). 
Nonetheless, the scope of these rural models needs to be sensitive to both the level of problems typically encountered in these areas and the quantity and quality of data that are generally available (Burkhardt et al. 1995; National Association of County Engineers 1995; King 1998).

Smerk (1978) suggests that sketch-planning techniques are highly appropriate for rural transit planning. These planning methods help analysts identify where multimodal opportunities exist, and pinpoint locations for more detailed study. Several sketch techniques or rules of thumb are available to perform this initial evaluation with minimal data requirements. Atkinson (1978) and Corradino (1978) present techniques for assessing the merits of local transit service in smaller communities. Both authors suggest use of the techniques to determine if threshold criteria are met and if further study is justified.

The Guidebook for Planning Small Urban and Rural Transportation Programs (U.S. DOT 1990) suggests that system-planning efforts for transit should focus on identifying need (number of people for which a service is targeted) rather than demand (number of anticipated trips by people in a market segment). In rural areas, the targeted market segments typically include elderly, handicapped, school-age children, and commuters to major job sites. The guidebook further suggests that service inventory and identification of service coordination opportunities should be part of the system-planning effort.

One of the more recent research efforts for estimating demand for rural passenger transportation was undertaken by Spielberg, Shaw, and McGetrick (1995) as part of the Transit Cooperative Research Program (TCRP) Project B-3. The authors reviewed dozens of previous transit-demand models as well as functional concepts for approaching demand estimation for both "program" and "nonprogram" demand-responsive transit services. Three consensus conclusions emerged from that work to guide development of a rural transit demand model:

1) The model should include variables for both market size and quantity of transit service to be made available.

2) The market for rural transit throughout the United States is largely composed of elderly, handicapped, and impoverished segments of the overall population. Rural transit is used by these segments largely for lifeline segments including work, medical care, and shopping trips. 
3) The model form should be based on the concept of decreasing returns to scale. In other words, while the per-person trip rate should increase with additional transit service, the rate of increase in the trip rate should decline as the amount of transit service continues to increase.

After careful comparison to existing rural transit operations in Georgia, the basic arguments of all these researchers were followed in developing an analysis process for the MTPT that would be suitable for system-level applications and would operate as a decision support, rather than decision-making, tool. Importantly, the arguments were adapted in a process that recognized the exclusive use of county-level, demand-responsive transit services in rural areas of Georgia.

\section{Transit Evaluation in the MTPT}

The basic focus of the MTPT transit analysis is to assess the feasibility for demand-responsive transit services. The tool was developed for application to new service rather than expansion or modification of existing services. The MTPT does not perform service planning, but rather assesses which counties merit consideration for initiating rural transit service. As such, the MTPT transit analysis is a decision-support tool that is intended to focus subsequent detailed service-planning efforts. Importantly, the MTPT transit analysis does not reach a conclusion about service feasibility; instead, it provides information that an analyst can use in reaching this decision.

In the MTPT, transit service feasibility considers three primary factors:

1) existence of human service transit providers in the county;

2) percentage of population in certain "target" populations; and

3)estimate of potential ridership, vehicle requirements, capital costs, operating costs, and economic benefits.

The first human service transit provider factor essentially notifies the analyst that opportunities may exist to coordinate service with existing providers, helping to address, in part, issues of funding for the operation of rural transit.

The second "target" population factor helps the analyst assess market characteristics of a geographic area through a comparison of county population statistics with both overall rural statewide averages for rural counties and 
statewide averages for counties with existing rural transit service. Ten key categories were consistently cited in the literature as major determinants of potential service need as opposed to demand.

The third "operational" factor provides a range of potential values for key operating and financial statistics. Based on user-selected values for level of service (operating hours and wait times) and type of transit vehicle, the MTPT estimates a range of values for the statistics using "off-the-shelf" analysis routines that have been customized for application to rural Georgia transit services.

The transit analysis is only functional in rural areas of the state (defined using the U.S. Census Bureau definition for nonurbanized areas). As mentioned earlier, the MTPT includes an urbanized county database of fully or partially urbanized counties. If a nonqualifying county is selected for analysis, the program returns error messages and truncates the transit analysis.

The MTPT includes a service provider database that identifies counties with existing transit services operated by a Federal Transit Administration (FTA) Section 5311-funded provider. The tool is not intended for use in analyzing service expansion or modification. Hence, if a county with current rural transit service is selected for analysis, the software will return an error message and cease subsequent evaluation. As can be seen, several avenues were followed to discourage inappropriate application of the transit routines.

\section{Service Coordination}

The MTPT first determines if rural transit in the analysis county is currently operated by a "human service" provider funded in whole or in part through the State of Georgia or FTA Section 5310 program. These human service providers operate transportation services for select groups of individuals who meet specific eligibility requirements (e.g., elderly). The services provided differ from general public transit services under FTA Section 5311. While nearly all human service transit providers in Georgia receive funding through the State of Georgia and FTA Section 5310, human service transit in other locations may be funded through other non-State and non-FTA sources.

In Georgia, a concerted effort is under way to find alternate means for providing transit services to these groups rather than having each human service 
provider purchase and operate its own vehicles. It is believed that alternate means of service provision, such as purchase of service from a regular rural transit agency, are cost efficient for the human service agency while providing an additional revenue stream to the regular rural transit provider. Hence, by identifying human service providers, a planner is notified that opportunities may exist to coordinate more extensive transit services that could have a more sustainable funding arrangement.

The service provider database included with the MTPT indicates counties in which these services are operated, service provider name, city where the provider is located, and funding source. If a human service provider is identified in the analysis area, a message is placed in the MTPT results report showing the name of the service provider and steps that an analyst should take to investigate the potential for service coordination.

\section{Service Need}

The second feasibility assessment category in the MTPT relates to service need. For the purposes of the analysis, service need is loosely defined as the number of people in different market segments for which a service is targeted. During MTPT development, an extensive literature review and outreach effort to rural transit planners and operators in Georgia identified 10 population segments that typically comprise the vast majority of the Georgia rural transit market:

- total persons age 60 and over;

- total persons living below poverty level;

- persons age 16 to 64 with mobility limitations;

- total persons with mobility limitations;

- employed persons with mobility limitations;

- persons enrolled in grade school;

- persons enrolled in high school;

- total households with no vehicles;

- persons using bus, walk, or bicycle modes to work; and

- persons using carpool to work.

These market segments are clearly not mutually exclusive. However, the service need analysis does not consider the absolute number of people in these 
different market segments, but rather their relative representation in the overall population of an analysis area. The MTPT provides the analyst with an estimate of the percentage of population in each market segment. The MTPT also provides two benchmark values for each market segment that allows the analyst to assess market characteristics of the target area:

- an average value for all Georgia rural counties; and

- an average value for all Georgia rural counties with existing public transit service.

The MTPT performs the service need assessment by querying an internal socioeconomic database that was developed from 1990 census data. While a variety of data sources were considered for this database, the Census Transportation Planning Package (CTPP) CD-ROM provides the data in the most accessible format. The poverty data were the only items that required estimation because the CTPP does not have a direct tabulation of impoverished households or individuals. The poverty estimation was generated using CTPP tables to estimate the number of households below the 1990 U.S. government poverty threshold and the number of persons per household. Population data are stored as raw numbers, and the MTPT calculates market percentages by dividing each socioeconomic category with total 1990 county population.

The socioeconomic database also includes land area and historical county population information. The historical data are used by the MTPT to estimate future values for total county population and portions of the market segments that are used to forecast service demand in subsequent analysis modules. Currently, the MTPT assumes that the relative percentage of total county population in each market segment does not change in the future. As part of an ongoing development of Georgia's statewide transportation plan, detailed countylevel population forecasts are being prepared that will include age and income estimates. It is anticipated that these population forecasts will be incorporated in the MTPT to enhance the service need and service demand components.

\section{Service Demand, Cost, and Benefits}

In the third part of the feasibility assessment, the MTPT estimates a range of potential values for key usage, equipment need, and cost statistics. Based on 
user-selected values for level of service (operating hours and wait times) and vehicle type, the MTPT provides estimates of annual ridership, fleet size, capital cost, annual operating cost, and annual economic benefit. The MTPT relies on "off-the-shelf" routines, including locally validated ridership equations, cost experience from Georgia operators, fleet estimation equations from other states, and a rural transit cost-benefit model developed for the GDOT. While many rural areas find that both social service (i.e., "program") and general public (i.e., "nonprogram") transit services are important, the MTPT only estimates demand, cost, and benefits for nonprogram services since the GDOT does not have funding and decision-making authority for program services.

Annual Ridership. The ridership routine provides an estimate of the annual number of transit trips per person based on the number of those individuals who are:

- age 60 and over,

- living in a household with annual wages below the poverty level, and

- age 16 to 64 and have a mobility limitation.

This transit rate per person is then multiplied by the population in these subgroups to arrive at an annual ridership estimate for the county. Estimates are provided for 2000 and 2010 using demographic data taken from the MTPT socioeconomic database that is used for the service need assessment. The MTPT provides a point estimate and range of likely values using the following equations:

Point Estimate: $Y=0.8983 * X^{0.822}$

Low Range: $\quad\left(-0.1073+(0.822 * \operatorname{LN}(X))-0.646 *\left[1.004+\left(\frac{(\operatorname{LN}(X)-1.17)^{2}}{359}\right)^{1 / 2}\right]\right)$

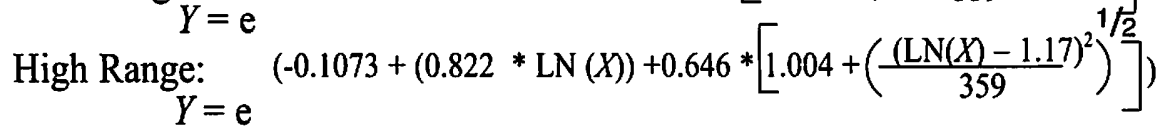

where:

$Y=$ annual trips per person within all targeted market segments (elderly, mobility limited, persons in poverty).

$X=$ annual transit revenue vehicle miles per capita.... 
In developing the ridership estimate, the MTPT user is allowed to select one of three typical levels of transit service corresponding to low (two revenue-vehicle miles [RVM] per capita), medium (five RVM per capita), and high (nine RVM per capita) service levels. These levels were identified based on current rural transit operations in Georgia. The service hours and availability that typically correspond to these levels are displayed in the MTPT dialogue box in Figure 1.

The ridership estimate is based on a methodology presented in TCRP Report 3 (Spielberg et al. 1995). This TCRP methodology was recalibrated for Georgia conditions to account for the exclusive use of county-based, demandresponsive service for general public transit in rural Georgia, and to improve overall model fit. The recalibration process is described in a subsequent section.

Fleet Size Requirements. Two separate equations, both taken from a rural transit analysis methodology developed for Florida (Corradino 1978), are used to estimate fleet size requirements. The first equation relies on the annual ridership previously described. The second equation relies on base RVM that are estimated by multiplying the service level selected above by total county population. Interestingly, both equations rely exclusively on operational parameters rather than vehicle size. The two estimates are averaged to arrive at a point

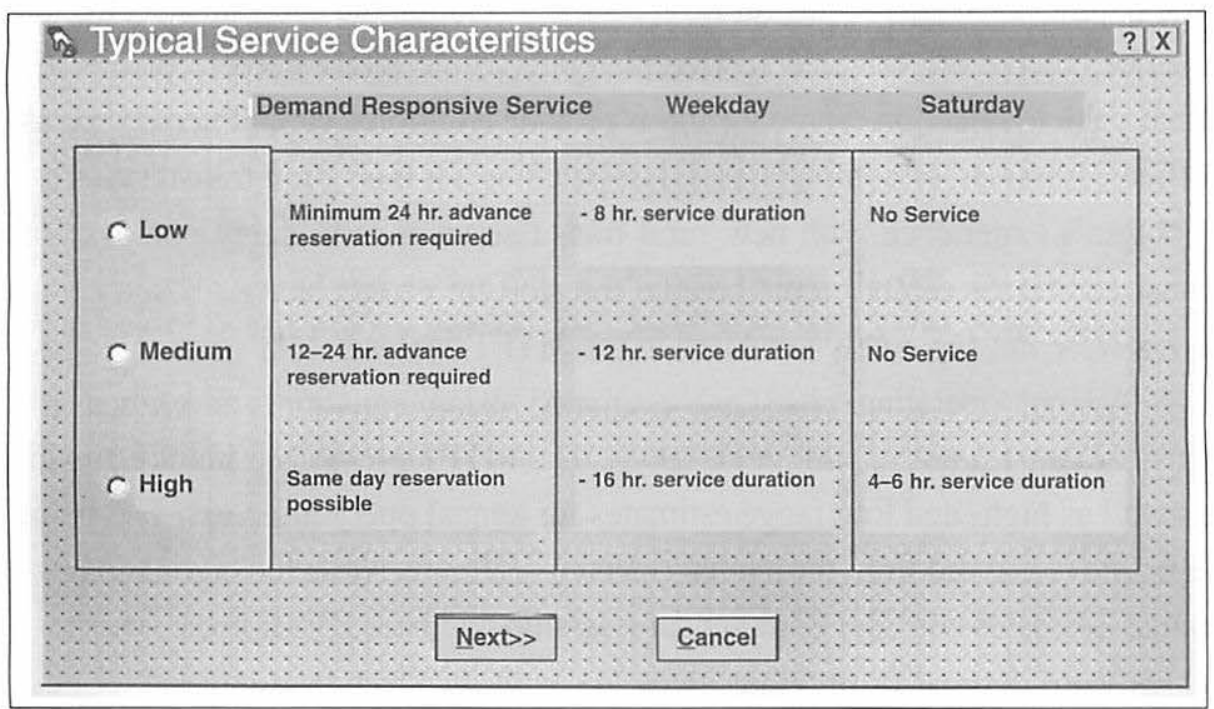

Figure 1. MTPT service-level dialogue box 
estimate for fleet size. A detailed test of these equations using recent operating data for Georgia's rural transit operators showed that an averaging of results from the two equations produced usable system-planning results. The specific equations used are:

$$
\begin{aligned}
& \text { Number of Vehicles }=\left(\frac{\text { Annual Demand }}{6,000}\right)^{\left(\frac{1}{1.8}\right)} \\
& \text { and } \\
& \text { Number of Vehicles }=\left(\frac{1.6 * \text { Base RVM }}{8,000}\right)^{\left(\frac{1}{1.7}\right)}
\end{aligned}
$$

Capital and Operating Costs. Capital costs are estimated in the MTPT by applying a unit cost to the fleet size estimate. Unit costs are based on recent GDOT acquisition experience for small and large buses, minibuses, and shutthe buses. Since these numbers are based on actual Georgia conditions, the values did not need additional adjustment. An MTPT user has the option of selecting different vehicle sizes in developing capital costs, as shown in the MTPT dialogue box example in Figure 2. In recent years, the GDOT has almost exclusively purchased small vans for rural transit. Although the user can select vehicles of other sizes, detailed service planning would likely need to justify the final vehicle selection. Some locations provide additional capital items such as office equipment or computer-based dispatching for their rural transit systems. Georgia's experience with new rural transit services is to delay purchase of most additional capital items beyond the start-up phase; hence, these capital costs were not separately tabulated in the MTPT analysis.

Annual operating costs are estimated using equations developed for TCRP Report 6 (Burkhardt et al. 1995). The MTPT provides a point estimate as well as high- and low-range estimates for annual operating costs. The point estimate is derived from the annual ridership estimate, while the high- and lowrange estimates consider both annual ridership and base RVM; these cost variables were derived in previous steps. As with the fleet size and capital cost methodologies, reviews of initial operating cost results using Georgia's recent 


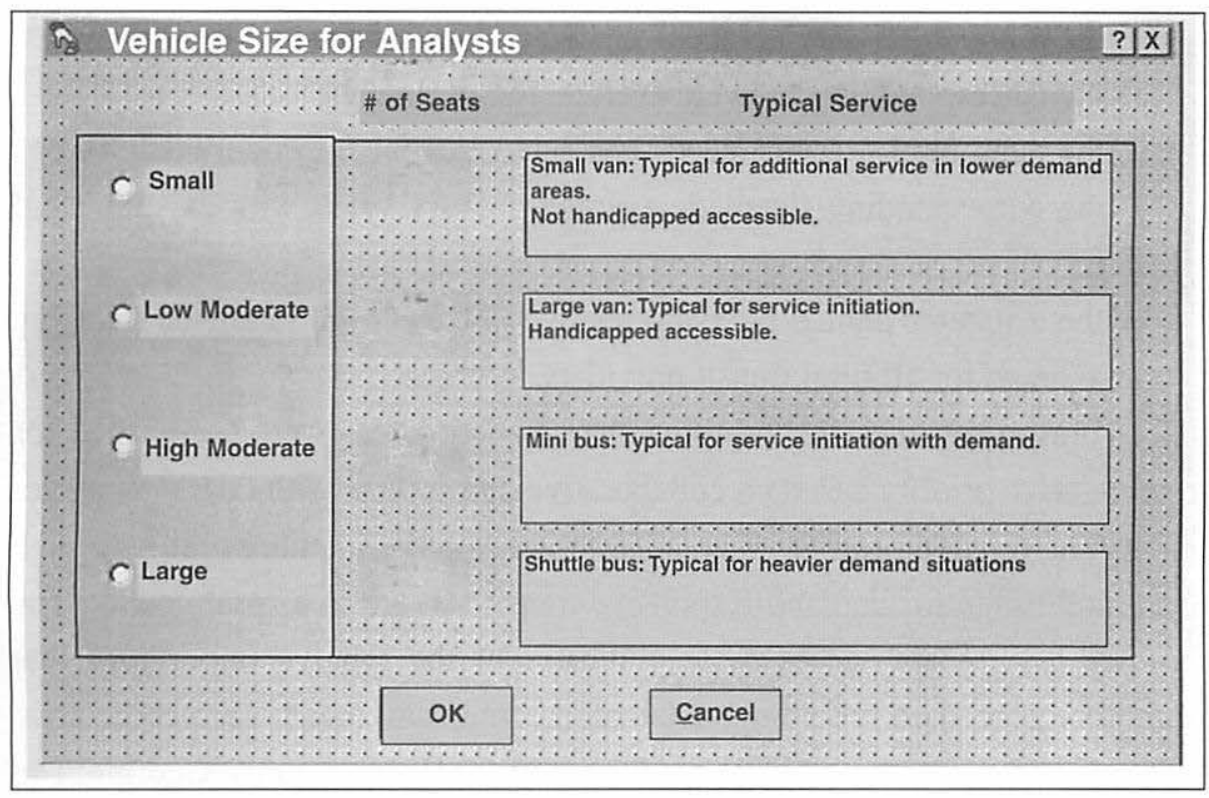

Figure 2. MTPT vehicle selection dialogue box

rural transit data showed a reasonable match between the estimated and actual costs for system-planning purposes.

Economic Benefits. The MTPT provides estimates of local economic impact and local government revenue impact based on the estimated average annual operating cost. The economic benefit estimate is based on relationships developed by the GDOT in the mid-1990s specifically for demand-responsive transit service in Georgia (Nelson and Peng 1998).

\section{Evaluation and Priority}

The MTPT was specifically structured so that the analyst will largely perform the evaluation and interpretation of the transit feasibility data. This structure was considered very important to discourage generic "black-box" applications of the system-level analysis. However, the MTPT does include a basic prioritization routine in the transit analysis to provide guidance to the user in interpreting the multiple data items estimated by the model.

The MTPT assigns a relative priority of either potential opportunity, moderate potential opportunity, high potential opportunity, or highest potential opportunity based on estimated characteristics in three areas: 
1) the number of "service-need" characteristics for the county that exceed the corresponding statewide average for all rural areas;

2) the number of "service-need" characteristics for the county that exceed the corresponding statewide average for rural areas with existing rural transit service; and

3)the estimated annual ridership relative to the current statewide ridership average for all rural transit providers.

Threshold values for the three characteristic areas were established for each relative priority based on collaborative discussions with GDOT management. The relative priority is intended to indicate the likelihood of both high need and usage for demand-responsive transit service in a rural county. The priority assignment serves as an indicator of the relative importance that should be placed on follow-up, detailed planning activities for a specific area. Figure 3 shows a sample MTPT results report for Bacon County, Georgia. The MTPT prioritized this rural county as having moderate potential opportunity for transit improvements based on demographic characteristics and estimated demand.

\section{Reestimation of Ridership Forecasting Routine}

The routine for forecasting rural transit ridership was adapted from TCRP Report 3. This method relies on RVM estimates and the number of people either with mobility impairments, living in poverty, or over age 60 , and was initially calibrated for the TCRP using a nationwide sample. Based on guidance in TCRP Report 3, the recommended level of application of this method was the county level for areas outside of Metropolitan Statistical Areas (MSAs) and with population densities under 1,000 persons per square mile. However, the authors expanded application of this method to all non-MSA counties in Georgia because a suitable estimation routine for demand-responsive service in higher-density areas could not be located.

Initial tests of this methodology on actual data from current rural transit providers in Georgia showed a large variation between predicted and actual ridership. When applied to Georgia, the TCRP method consistently overestimated ridership when transit service provision was low, and underestimated 


\section{TRANSIT MODE ANALYSIS}

The Program has determined that transit services in Bacon County are operated by the following social service transportation providers:

ALMA -- BACON COUNTY MR SERVICE PROVIDER

Comparison of Socioeconomic Characteristics For Bacon County

Column $A$ is the average for Bacon county

Column $B$ is the average of all rural counties

Column $C$ is the average of all rural counties with transit service

Data Category

Total Persons aged 60 and over

Total Persons living below poverty level

Persons aged 16 to 64 with mobility limitations

Persons enrolled in grade school

Persons enrolled in high school

Total persons with mobility limitations

Employed persons with mobility limitations

Total households with no vehicles

Persons using bus, walk or cycle modes to work

Persons using carpool to work
A

$\begin{array}{lll}16.97 & 16.14 & 16.13 \\ 09.94 & 17.04 & 18.40 \\ 08.22 & 01.90 & 01.98 \\ 15.08 & 14.24 & 14.02 \\ 05.49 & 04.27 & 04.44 \\ 11.16 & 04.68 & 04.73 \\ 00.41 & 00.31 & 00.31 \\ 11.20 & 09.60 & 09.08 \\ 00.95 & 01.31 & 01.20 \\ 08.46 & 08.24 & 08.50\end{array}$

*** Values are expressed as percent of total population. ***

Based on the selected characteristics summarized above, the following values have been estimated for transit service in Bacon county:

* Annual demand may be between 2,740 and 10,370 with a mean value of 5,330 for 1990.

* Annual demand may be between 2,870 and 10,850 with a mean value of 5,580 for 2000.

* Annual demand may be between 3,000 and 11,350 with a mean value of 5,830 for 2010.

* Vehicle requirements are estimated between 2 and 3 for full start-up.

* Capital costs are of the magnitude of (1997) $\$ 467,500$ for full start-up.

* Annual operating costs in the range of (1997) $\$ 8,600$ to (1997) $\$ 96,420$ with a mean value of (1997) $\$ 33,780$.

* The annual local economic impact from operating transit service is estimated to be in the range:

1. Local Economic Impact: $\$ 939,460$ to $\$ 1,699,550$

2. Local Government Revenue Impact: $\$ 11,820$ to $\$ 71,610$

* Bacon County is classified as a county with a priority classification as Moderate Potential Opportunity for Transit Improvements.

Figure 3. Sample MTPT transit output 
ridership when transit service provision was moderate. It was thought that these estimation problems could have arisen from: (1) a preponderance of demand-responsive service in Georgia as compared to other states, (2) overall lower levels of transit service levels as compared to other states, or (3) expansion of the methodology into locations beyond the intended area of application.

Therefore, the model was recalibrated using data only for Georgia's rural public transit operators.

The discussion earlier in this article of rural transit planning noted that the model form for ridership forecasts should be based on the concept of decreasing returns to scale. In other words, while the per-person trip rate should increase with additional transit service, the rate of increase in the trip rate should decline as the amount of transit service continues to increase. This essentially means that the model should be asymptotic toward a fixed-trip rate. However, investigation of the actual equations provided in TCRP Report 3 indicated that this model form was not used. Instead, the equations exhibited an increasing returns to scale rather than a decreasing returns to scale. Further analysis suggested that this model form (i.e., increasing returns to scale) accounted for much of the prediction error for Georgia conditions.

The ridership equations were reestimated using calibration and validation datasets developed from eight years of operating statistics for Georgia's rural transit operators and the MTPT socioeconomic database. An initial analysis of census data suggested that use of the "static" MTPT socioeconomic database was acceptable since changes in the rural population subsegments tended to occur very slowly, and overall growth rates in rural regions are very low. Other initial tests on different model forms also indicated that a "decreasing returns to scale" model form using RVM (rather than other operational variables) would produce the best statistical results.

The recalibrated model was then evaluated with the validation dataset. Three key statistics (average absolute mean difference, correlation coefficient, and $r$-squared) were calculated for the observed and predicted values of the validation dataset. Overall, the validation statistics, as shown in Table 1, indicated that the reestimated model performed reasonably well in predicting trip 
rates, but resulting confidence intervals might still be somewhat larger than desired due to the remaining spread in observed trip rates in both the calibration and validation datasets.

\begin{tabular}{|l|c|}
\hline \multicolumn{2}{|c|}{ Table 1 } \\
\multicolumn{2}{|c|}{ Validation Test Results for Recalibrated Model } \\
\hline Average annual observed trip rate for market segments & Value \\
Average absolute mean difference in trip rate & 3.66 \\
$R$-square (validation dataset using calibrated model) & 1.59 \\
$R$-square (calibration datset) & 0.65 \\
Correlation coefficient (observed versus predicted for validation dataset) & 0.70 \\
\end{tabular}

The effect of this spread was reviewed to establish appropriate prediction intervals for the newly calibrated model. Several potential confidence levels were evaluated to determine an acceptable level of confidence. In general, the selection of a specific percentile value for a confidence or prediction interval is guided by the decision-making need that the resulting information is intended to serve. Less sensitive decisions, such as those of a preliminary nature used to guide further studies, can be based on information in which less confidence exists. Since the MTPT transit results are intended as an initial assessment of potential transit suitability, a 70 percent confidence level was identified as adequate.

\section{Potential Enhancements}

The previous sections have described a process for initially analyzing the suitability of rural counties for demand-responsive transit service. The entire MTPT tool, including the transit analysis, is still undergoing a comprehensive evaluation by transportation planners at state and local agencies throughout Georgia. It is expected that this review will uncover ways to improve the MTPT in terms of both functionality and user-friendliness. The authors have identified a preliminary list of areas in which the MTPT transit analysis routine could be enhanced in the coming years:

- Revise the entire socioeconomic database upon receipt of results from the year 2000 U.S. census. 
- Test the effect of different service characteristics on model form. This test would involve providing a more precise statistical match between service characteristics (days and hours of operation, advance reservation requirements, etc.) and the RVM/capita input to the demand model. This option would require stratifying the input dataset based on these service characteristics.

- Test if the three market segments (elderly, impoverished, and handicapped) are individually significant for the ridership model or if there is significant overlap in the population groups; the three market segments are considered collectively in the current model. This option could require collection of detailed ridership data in some test locations.

- Test if other market segments, such as junior and senior high school populations, and special generators, such as colleges or resort areas, could be significant predictors of transit demand in Georgia.

- Test if more complex model forms, such as a logit model, would produce significantly better ridership forecasts.

- Revalidate the fleet size equations to test if service area is a significant factor in determining fleet size requirements.

- Provide increased MTPT user flexibility by permitting the program user to override default vehicle costs currently assigned within the program.

\section{Summary and Conclusions}

The ability for a governing agency to perform a wide-scale analysis for rural transit using a robust computer analysis tool affords policymakers the benefit of impartially identifying prospective regions where rural transit implementation will conceivably afford the greatest benefits. The MTPT transit module is such a tool and serves as an initial indicator for helping analysts with decisions regarding the type and extent of rural transit service needs. The balance of transit service coordination, need, and demand offered by the MTPT supports all initial aspects of rural transit analysis. The methods used by the tool incorporate nationally accepted standards that are modified to adapt to conditions unique to the State of Georgia. The tool filters nonqualifying regions from analysis, and prioritizes prospective transit services for qualifying rural areas. 
Though a single analysis tool should not be used to justify final policy and implementation decisions, the MTPT transit module serves as a solid foundation for the ultimate rural transit analysis and decision structure for the State of Georgia.

\section{References}

Atkinson, Wallace. 1978. Estimating transit demand, fleet size and costs for small communities. NCHRP Special Report 187: 29-33.

Attaluri, P., M. Javid, and P. N. Seneviratne. 1997. Modeling demand for public transit services in rural areas. Journal of Transportation Engineering 123 (3).

Black W. R. 1993. Development of IDEAL model for identification of rural public transit needs. Transportation Research Record 1402: 107-109.

Burkhardt, J. E., B. Hamby, and A. T. McGavock. 1995. Users manual for assessing service-delivery systems for rural passenger transportation. Transit Cooperative Research Program Report 6.

Corradino, Joseph. 1978. Forecasting transit ridership in smaller communities. NCHRP Special Report 187: 24-29.

Dixon, Karen K., Wayne Sarasua, Janice Daniel, and George D. Mazur. 2000. A tool for rural and statewide multimodal transportation planning. Journal of Computing in Civil Engineering. Forthcoming.

Federal Highway Administration (FHWA). 1995. General observations on statewide transportation planning. Unpublished agency memorandum. Washington, DC.

Fleet, Christopher. 1978. Achieving a balance between long-range and short-range planning at an appropriate level of effort. NCHRP Special Report 187: 11-16.

Georgia Department of Transportation (GDOT). 1994. Draft statewide transportation plan. Atlanta, GA.

Golenburg, Marvin. 1978. Procedure for analysis of system sufficiency and deficiency (abridgment). NCHRP Special Report 187: 85.

King, D. 1998. What are the "best practices" of rural subarea planning? Report FHWA-AZ-98-469. Washington, DC: Federal Highway Administration.

Litz, Leon E. 1980. Measuring highway improvement needs and priority analysis. Federal Highway Administration. Washington, DC.

Mazur, George, and Wayne Sarasua. 1997. Georgia's rural transportation planning tool: Something old, something new.... Presented at the Sixth TRB Conference on the Application of Transportation Planning Methods. Dearborn, MI.

Mazur, George, Wayne Sarasua, and Janice Daniel. 1996. A multimodal transportation planning tool for rural areas in Georgia. Transportation Research Record 1552: $48-56$. 
National Association of County Engineers. 1995. Rural transportation planning, 1995 Edition, NACE Action Guide. Washington, DC: Federal Highway Administration. Nelson, A. C., and Z. Peng. 1998. Rural transit service: A local economic and fiscal impact analysis. Transportation Research Record 1623: 57-62.

Smerk, George. 1978. Transportation and transit planning for small- and mediumsized urban areas. NCHRP Special Report 187: 18-21.

Smith, J. D. 1982. The choice of appraisal techniques when resources are limited-A case study of the rural roads programme in peninsular Malaysia. Highway Development in Developing Countries. Proceedings of the Conference on Criteria for Planning Highway Investment in Developing Countries: 71-77.

Spielberg, Frank, Gordon Shaw, and Christie McGetrick. 1995. Workbook for estimating demand for rural passenger transportation. Transit Cooperative Research Program Report 3.

Stover, Vergil. 1978. Appropriate level of effort for planning for small- and mediumsized communities. NCHRP Special Report 187: 9-11.

U.S. Department of Transportation. 1990. Guidebook for planning small urban and rural transportation programs-Volume 1. Washington, DC: New Mexico State Highway and Transportation Department.

\section{About the Authors}

George Mazur (gdm@camsys.com) is an associate with Cambridge Systematics, based out of the CS Oakland, California, office. He has 12 years of experience in transportation planning and policy, environmental analysis, and analytical tool development and application. He has civil engineering degrees from Purdue University and the University of California at Berkeley. Mr. Mazur is a registered Professional Engineer in California and Georgia.

DR. KaREN Dixon, P.E. (karen.dixon@ce.gatech.edu), is an assistant professor in the School of Civil and Environmental Engineering at the Georgia Institute of Technology. She received civil engineering degrees from North Carolina State University and Texas A\&M University. Dr. Dixon focuses her research in the areas of transportation design, operations, and safety.

DR. WAYNe A. SARASUa (sarasua@clemson.edu) is an assistant professor in the Department of Civil Engineering at Clemson University. Dr. Sarasua's research and teaching interests center on the application of geographic information system technologies to transportation. 